\title{
Carbon Nanotubes: Properties and Medical Applications
}

\author{
Ibrahim H. Alfahdawi ${ }^{1}$ and Wasan Mohammed Alsewidi ${ }^{2 *}$ \\ ${ }^{1}$ Department of Dentistry, Al-Farabi University College, Baghdad, Iraq. \\ ${ }^{2}$ Alfarabi University College, Department of Biology, Baghdad, Iraq.
}

*Correspondence Author: Wasan Mohammed Alsewidi, Alfarabi University College, Department of Biology, Baghdad, Iraq.

Received date: January 28, 2021; Accepted date: March 09, 2021; Published date: March 20,2021

Citation: Ibrahim H. Alfahdawi and Wasan M. Alsewidi, (2021) Carbon Nanotubes: Properties and Medical Applications. Clinical Medical Reviews and Reports 3(3); DOI:10.31579/2690-8794/061

Copyright:@2021 Wasan Mohammed Alsewidi, This is an open access article distributed under the Creative Commons Attribution License, which permits unrestricted use, distribution, and reproduction in any medium, provided the original work is properly cited.

\begin{abstract} explored, in the last few years, for applications in biology and biomedicine.

Keywords: carbon based nanomaterial;biomedical applications; nano-technology
\end{abstract}

Since the beginning of the twenty-one century, carbon based nanomaterial (CNTs) has been introduced in (pharmacy and medicine) in therapeutic for system of drug delivery. NTs have proved able to transport a wide range of molecules across membranes and into living cells; therefore, they have attracted great interest in biomedical applications such as advanced imaging, tissue regeneration, and drug or gene deliver. As it is known, carbon nanotubes exhibit various unique intrinsic chemical and physical properties and it has been intensive

\section{Introduction}

Recently, many researches have focused on nano-technology and nanocomposites. Researchers select specific filler and introduce it into a specific polymer to achieve a specific composite with modified properties. If the filler is a nano material, the composite is termed a nanocomposite. Nanotechnology brings evolutionary changes to everyday life [1]. Nanotechnology has opened a window for the development of diverse organic and inorganic drug carriers, known as nanoparticles. The greatest advantage is its potential to create novel structures with enhanced abilities to translocate through cell membranes, and increased solubilization, stability, and bioavailability of biomolecules, thereby enhancing their delivery efficiency [2-4].

\section{Nanotechnology}

The word "nano" is from the Greek, meaning dwarf (small); scientific treatment at the nano level (atomic level) with the help of special scientific instruments is known as nanotechnology, which has become a wellknown field in the last three decades. Norio Taniguchi coined the word "nanotechnology" in 1974, in Japan. He stated that, "nanotechnology mainly consists of the processing steps of the separation, consolidation and deformation of materials by one atom or one molecule. Nanotechnology is a vast field which explores many facts about the structures and properties of materials [5, 6]. Nanomaterials have sizes ranging from about one nanometer up to several hundred nanometers, comparable to many biological macromolecules such as enzymes, antibodies, DNA plasmids, etc. Materials in this size range exhibit interesting physical properties, distinct from both the molecular and bulk scales, presenting new opportunities for biomedical research and applications in various areas including biology and medicine. As an emerging field, nanobiotechnology bridges the physical sciences with biological sciences via chemical methods in developing novel tools and platforms for understanding biological systems and disease diagnosis and treatment [7, 8 and 9]. Nanoparticles are generally $<1000 \mathrm{~nm}$ in at least one dimension, and consist of different biodegradable materials such as natural or synthetic polymers, lipids, or metals as phospholipids, lactic acid, chitosan, dextran, polyethylene glycol (PEG), cholesterol, carbon, silica [10].

\section{Carbon Nanotubes (CNTs)}

Carbon nanotubes (CNT) are a group of nanomaterials consisting of cylindrical hollow carbon based fibers (tubes). They have very high aspect ratios with diameters lower than about $100 \mathrm{~nm}(0.000001 \mathrm{~mm})$ and lengths that can reach several hundred micrometers. They can be produced or refined to possess specific properties such as high electrical conductivity, optical transmission, or extremely high tensile strength. They are already used in several existing products in e.g., gas and energy storage, electronics, sensors, optics, and as additives in various nanocomposite materials. It is important to note that crude CNT are usually inhomogeneous and have a high abundance of carbon and inorganic catalyst impurities. Due to technical challenges, commercial products are rarely fully purified. The actual purity and CNT quality depends on the manufacturing procedure and type of potential refinement of the products [11].

Conceptually the nanotubes are viewed as rolledup structures of single or multiple sheets of graphene to give SWCNTs and MWCNTs, respectively. These onedimensional carbon allotropes are of high surface area, high mechanical strength but ultra-light weight, rich electronic properties, and excellent chemical and thermal stability. Ever since the discovery of carbon nanotubes, researchers have been exploring their potential in biological and biomedical applications [12].

\section{Structure of Carbon Nanotubes}

CNT are typically grouped according to number of sidewalls and the atomic structure in the sidewalls. The simplest CNT are single-walled 
(SW), double-walled (DW) and multi-walled (MW) CNT. It is relatively easy to imagine a single-wall carbon nanotube (SWNT). Ideally, it is enough to consider a perfect graphene sheet (graphene is a polyaromatic monoatomic layer consisting of sp2-hybridized carbon atoms arranged in hexagons; genuine graphite consists of layers of this graphene) and to roll it into a cylinder (Fig. 1), making sure that the hexagonal rings placed in contact join coherently. Then the tips of the tube are sealed by two caps, each cap being a hemi-fullerene of the appropriate diameter (Fig. 2a-c) [13].

Few to a few tens of concentric cylinders with the regular periodic interlayer spacing locate around ordinary central hollow and made MWCNTs. The real space analysis of multiwall nanotube images has shown a range of interlayer spacing $(0.34$ to $0.39 \mathrm{~nm})$. Depending on the number of layers, the inner diameter of MWCNTs diverges from $0.4 \mathrm{~nm}$ up to a few nanometers and outer diameter varies characteristically from two $\mathrm{nm}$ up to 20 to $30 \mathrm{~nm}$. Both tips of MWCNT usually have closed and the ends are capped by domeshaped half-fullerene molecules (pentagonal defects), and axial size differs from one $\mu \mathrm{m}$ up to a few centimeter. The role of the half-fullerene molecules (pentagonal ring defect) is to help in closing of the tube at the two ends [14]. On other hand, SWCNT diameters differ from 0.4 to 2 to $3 \mathrm{~nm}$, and their length is typically of the micrometer range. SWCNTs usually can come together and form bundles (ropes). In a bundle structure, SWCNTs are hexagonally organized to form a crystallike construction [15].
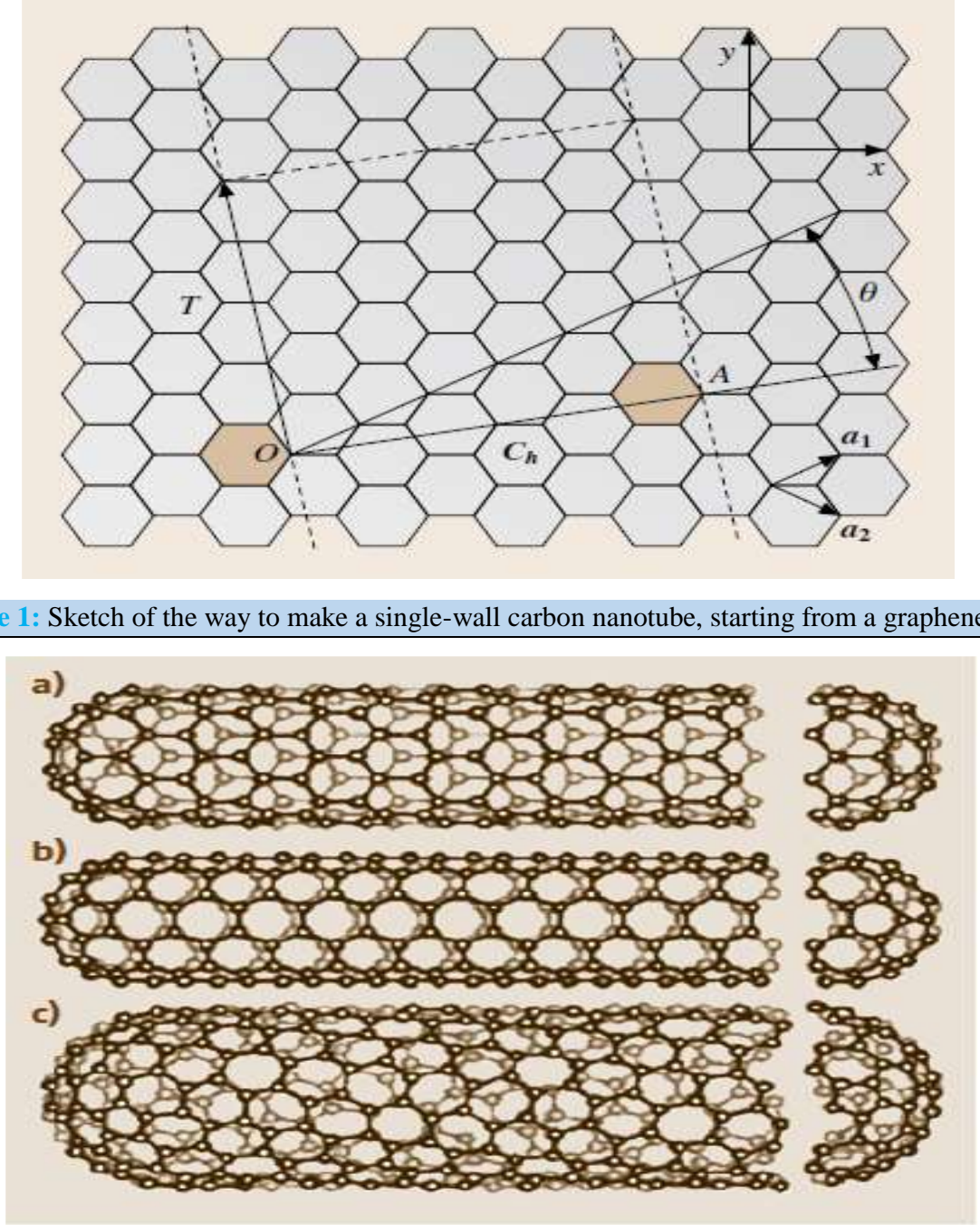

Figure 2: a-c Sketches of three different SWNT structures that are examples of (a) a zigzag-type nanotube, (b) an armchair-type nanotube, (c) a helical nanotube

\subsection{Single-Wall Nanotubes}

Geometrically, there is no restriction on the tube diameter. However, calculations have shown that collapsing the single-wall tube into a flattened two-layer ribbon is energetically more favorable than maintaining the tubular morphology beyond a diameter value of $\approx 2.5 \mathrm{~nm}$. On the other hand, it is easy to grasp intuitively that the shorter the radius of curvature, the higher the stress and the energetic cost, although SWNTs with diameters as low as $0.4 \mathrm{~nm}$ have been synthesized successfully. A suitable energetic compromise is therefore reached for $\approx 1.4 \mathrm{~nm}$, the most frequent diameter encountered regardless of the synthesis technique (at least for those based on solid carbon sources) when conditions ensuring high SWNT yields are used. There is no such restriction on the nanotube length, which only depends on the limitations of the preparation method and the specific conditions used for the synthesis (thermal gradients, residence time and so on). Experimental data are consistent with these statements, since SWNTs wider than $2.5 \mathrm{~nm}$ are only rarely reported in the literature, whatever the preparation method, while the length of the SWNTs can be in the micrometer or the millimeter range. These features make single-wall carbon nanotubes a unique example of single molecules with huge aspect ratios [16]. 


\subsection{Double- and Multiple-Walled Carbon Nanotubes}

DWNTs and MWNTs may be understood as two and multiple concentric SWNTs, respectively [17]. Multi-walled carbon nanotubes (MWCNTs) are built from several graphenes and, because of this, describing them does not require considering morphology and structure only, as for SWCNT, but also texture and nanotexture. MWCNTs may be defined as nanosized, hollow, carbon filaments whose wall is made up with more than one graphene, with the intergraphene distance being equal to the regular $\sim 0.34 \mathrm{~nm}$ van der Waals distance for turbostratic, polyaromatic carbons (see the following). However, this definition is not consensual. It is often considered in the literature that the term 'MWCNT' should be restricted to the concentric assembly of a series of $n$ SWCNTs (with $n \geq$ 2 , and no limitation for the upper value) with increasing diameter (Fig. 3a) which corresponds to the typical texture of arc-grown MWCNTs which were shown for the first time in the 1991 Minima's landmark paper. In such a case, any other carbon nanofilament, hollow or not, specifically those exhibiting the so-called 'herringbone' texture where the graphenes making the wall are oblique (Fig. $3 \mathrm{~b}$ and c) and sometimes transverse with respect to the tube axis, is preferably named carbon nanofibre (CNF). We will not adopt such a definition line here. We prefer to follow the reasonable opinion that, provided it is made of carbon, hollow, with a nanosized diameter (i.e. $<100 \mathrm{~nm}$ ), and with a wall made up with an assembly of several graphenes, a filament may be named 'MWCNT', regardless of the orientation of the graphenes making the tube wall with respect to the tube axis (in this regard, the 'multi-wall' denomination is not really appropriate and 'multi-graphene wall' would have been more suitable, but the use of the former is now too much widespread). We thus consider that carbon nanofilaments divide into carbon nanotubes and carbon nanofibres. The latter do not exhibit any empty core, whereas the former are hollow, whether the inner cavity is continuous or not, that is, whether it is incidentally or periodically obstructed with graphenes oriented transversally with respect to the tube axis [18].

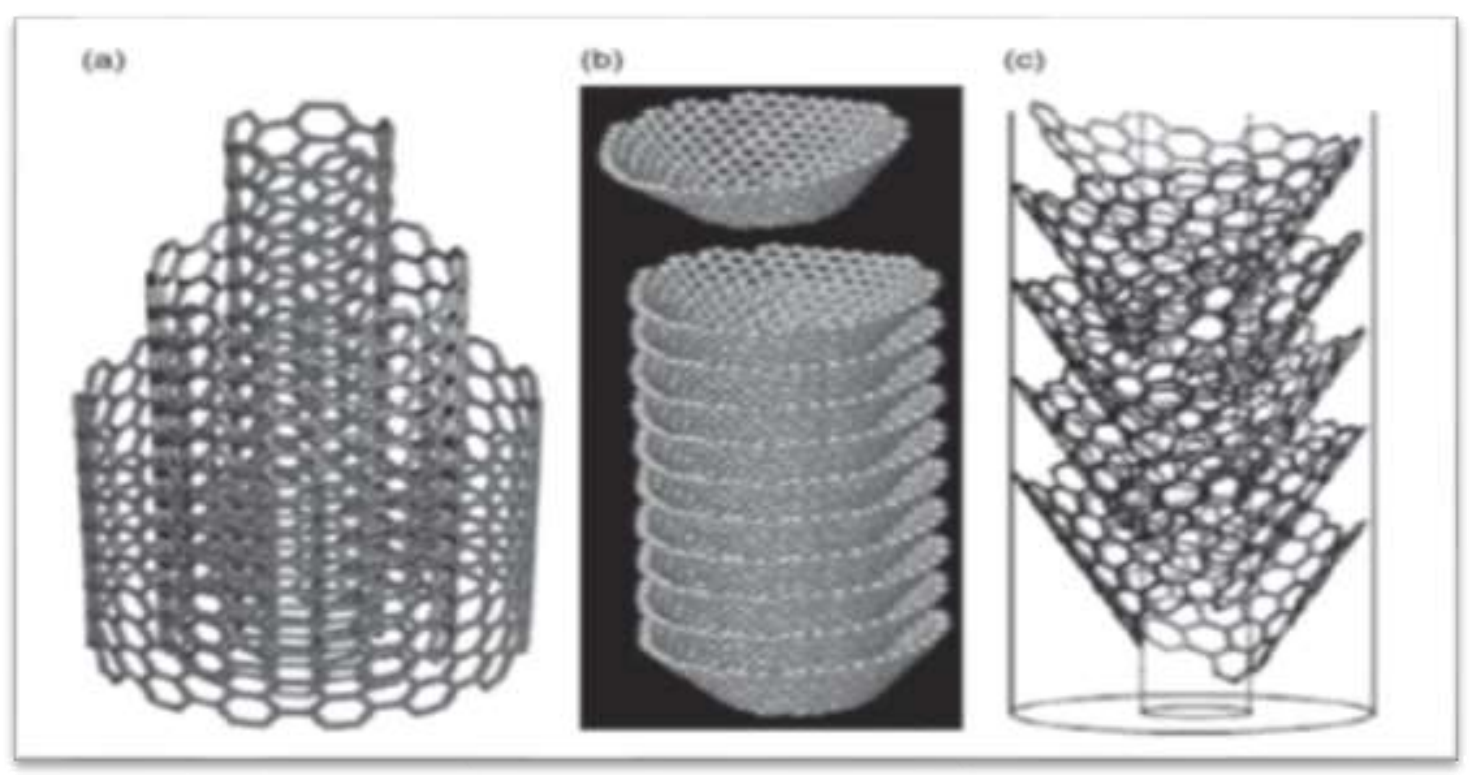

Figure 3: (a) Model of a so-called 'concentric' texture. (b) Model of a so-called 'herringbone' texture, for which truncated graphene cones are piled up as cups (cup-stake model, by courtesy of M. Endo). (c) Another model for the herringbone texture, for which a single graphene ribbon is helically wrapped.

\section{Strength}

Carbon nanotubes have a higher tensile strength than steel and Kevlar. Their strength comes from the $\mathrm{sp}^{2}$ bonds between the individual carbon atoms. This bond is even stronger than the $\mathrm{sp}^{3}$ bond found in diamond. Under high pressure, individual nanotubes can bond together, trading some $\mathrm{sp}^{2}$ bonds for $\mathrm{sp}^{3}$ bonds. This gives the possibility of producing long nanotube wires. Carbon nanotubes are not only strong, they are also elastic. You can press on the tip of a nanotube and cause it to bend without damaging to the nanotube and the nanotube will return to its original shape when the force is removed. A nanotube's elasticity does have a limit, and under very strong forces, it is possible to permanently deform to shape of a nanotube. A nanotube's strength can be weakened by defects in the structure of the nanotube. Defects occur from atomic vacancies or a rearrangement of the carbon bonds. Defects in the structure can cause a small segment of the nanotube to become weaker, which in turn causes the tensile strength of the entire nanotube to weaken. The tensile strength of a nanotube depends on the strength of the weakest segment in the tube similar to the way the strength of a chain depends on the weakest link in the chain [19].

\subsection{Electrical Properties of CNTs}

As mentioned previously, the structure of a carbon nanotube determines how conductive the nanotube is. When the structure of atoms in a carbon nanotube minimizes the collisions between conduction electrons and atoms, a carbon nanotube is highly conductive. The strong bonds between carbon atoms also allow carbon nanotubes to withstand higher electric currents than copper. Electron transport occurs only along the axis of the tube. Single walled nanotubes can route electrical signals at speeds up to $10 \mathrm{GHz}$ when used as interconnects on semi-conducting devices. Nanotubes also have a constant resistively [19].

\subsection{Thermal Properties of CNTs}

The strength of the atomic bonds in carbon nanotubes allows them to withstand high temperatures. Because of the carbon nanotubes have been shown to be very good thermal conductors. When compared to copper wires, which are commonly used as thermal conductors, the carbon nanotubes can transmit over 15 times the amount of watts per meter per Kelvin. The thermal conductivity of carbon nanotubes is dependent on the temperature of the tubes and the outside environment [20].

\section{Properties}




\subsection{Mechanical Properties of CNTs}

CNTs are considering as the strongest materials in nature at this point. The literature suggests that CNTs are very strong materials, especially in the axial direction. The Young's modulus ranges from 270 to $950 \mathrm{GPa}$, while the tensile strength is also very high, in the range of 11-63 GPa. Several reports have shown that in the radial direction, CNTs are rather soft [21]. The first TEM observation of radial elasticity showed that Vander Waal's forces could deform two adjacent nanotubes [22]. Later, different groups of researchers to measure the radial elasticity of MWNTs quantitatively performed nano-indentations with an atomic force microscope (AFM), and tapping/contact mode AFM was recently used to examine SWNTs $[23,24]$. The results showed that CNTs are in fact very soft in the radial direction. The radial direction elasticity of CNTs is important, especially for the formation of CNT nanocomposites and their mechanical properties, in which embedded tubes are subjected to large deformation in the transverse direction when a load is applied to a composite structure. Because the carbon-carbon bonds observed in graphite are among the strongest in nature, CNTs have excellent potential, as they are the stiffest and toughest structure ever synthesized by scientists. Studying CNTs under TEM has shown that these materials are flexible and do not break upon bending [25]. Earlier theoretical calculations were carried out to predict the mechanical properties of CNTs. The first attempt to determine the Young's modulus for individual MWNTs was performed by Treacy et al., who measured the amplitudes of thermal vibrations in CNTs under TEM. They demonstrated that nanotubes possess an average Young's modulus of $\mathrm{Y}=1-1.8 \mathrm{TPa}$, which is extremely high compared to commercially available carbon fibers ( $\mathrm{Y} \geq$ $800 \mathrm{GPa}$ ). Various research groups performed direct measurements of the bending forces of MWNTs as a function of the displacement inside an AFM. The values for the Young's modulus were found to be between 0.32 and $1.47 \mathrm{TPa}[26,27,28,29]$.

Falvo et al. [27], observed that MWNTs could be bent at sharp angles without undergoing any structural fracturing using an AFM tip. Endo et al. observed that while breaking vapor-grown CNTs in liquid nitrogen, an inner tubule could survive this pressure. Zhu et al. [30], applied high pressures $(50 \mathrm{GPa})$ at room temperature, using shock waves, to arcdischarge MWNTs and noted that the tubes do not break but collapse; that is, the outer shells transform into curled graphene domains while the inner cores display structural defects. Sinnot et al. [31], who found that SWNTs could exhibit a Young's modulus as high as that of diamond, carried out more theoretical work on the mechanical properties of CNTs. Yakobson and $\mathrm{Ru}$, proposed a mechanism of CNT transformation under uniaxial tension, leading to pentagon-heptagon defects in these tubes under high stress [32, 33]. Guanghua et al., also showed theoretically that the mechanical properties of SWNTs are mainly dependent on the diameter. They estimated the theoretical Young's modulus of nanotubes, of »one $\mathrm{nm}$ diameter is in the range of 0.6-0.7 TPa [34].

Hernandez et al., also obtained theoretical values of the Young's modulus that were in close agreement with those obtained experimentally for MWNTs (1-1.2 TPa). They also reported that the increase in diameter is proportional to the enhancement of the mechanical properties and that if the diameter is increased to a certain value, the Young`s moduli of the tubes approach those of planar graphite [35].

\section{Applications of CNTs}

Given the increased profits associated with medical technology related to gene therapy, cancer treatments, and innovative new answers for lifethreatening diseases, the science of nanomedicine has become the most rapidly growing field. The unique properties and characteristics of CNTs enable scientists to develop new areas in nanomedicine. SWNTs and MWNTs have already proven their potential to serve as safer and effective alternatives to previous drug delivery methods. They can pass through membranes, carrying therapeutic drugs, vaccines, and nucleic acids deep into the cell to the substrate targets. They serve as ideal non-toxic vehicles, which in some cases; increase the solubility of the drug, resulting in greater efficiency and safety. Overall, recent studies of CNTs have shown a very promising future for them in medicine [36]. The properties of nanotubes are certainly amazing; in the last few years, many studies have suggested potential applications of CNTs and have shown innumerable applications that could be promising when these newly determined materials are combined with typical products [37, 38]. Production of nanorods using CNTs as reacting templates [39, 40, 41, 42]. Applications for nanotubes encompass many fields and disciplines such as medicine, nanotechnology, manufacturing, construction, electronics, and so on. The following application can be noted: high-strength composites, actuators, energy storage and energy conversion devices, nanoprobes and sensors, hydrogen storage media, electronic devices, and catalysis [43, 44, 45, 46]. However, the following sections detail existing applications of CNTs in the biomedical industry exclusively. Before use of carbon nanotube in biological and biomedical environments, there are three barriers, which must be overcome: functionalization, pharmacology, and toxicity of CNTs. One of the main disadvantages of carbon nanotubes is the lack of solubility in aqueous media, and to overcome this problem, scientists have been modifying the surface of CNTs, i.e., fictionalization with different hydrophilic molecules and chemistries that improve the water solubility and biocompatibility of CNT [47]. Another barrier with carbon nanotube is the biodistribution and pharmacokinetics of nanoparticles that are affected by many physicochemical characteristics such as shape, size, chemical composition, aggregation, solubility surface, and fictionalization. Studies have shown that water-soluble CNTs are biocompatible with the body fluids and do not any toxic side effects or mortality. Another important barrier is toxicity of CNTs. Generally, the combination of the high surface area and the intrinsic toxicity of the surface can be responsible for the harmful effects of nanoparticles [48].

\section{Medical Applications of CNTs}

Since the beginning of the 21st century, CNTs have been introduced in pharmacy and medicine for drug delivery system in therapeutics. A number of biomedical applications of CNTs are proposed including drug vectors, biomolecule, gene delivery to cells or organs, tissue regeneration, and biosensor diagnostics and analysis [49]. In fact, due to their high surface area, excellent chemical stability, and rich electronic polyaromatic structure, CNTs are able to adsorb or conjugate with a wide variety of therapeutic molecules (drugs, proteins, antibodies, DNA, enzymes, etc.) and to carry them near the targeted cell. The drug can either be loaded into the CNT structure or be attached to the CNT surface. Either two different systems of drug delivery conjugates with CNT have been described without internalization of the CNT carrier or both the drug and the CNT carrier can enter the cells either via the endocytosis pathway or via the insertion and diffusion pathway. The internalization method is more effective than surface attachment. In fact, in the former method, after entering the cells, the intracellular environment degrades the conjugated drug releasing pharmacological agents inside the cells, while in the surface attachment method; the drug can be degraded in the physiological fluids prior to be internalized by the cells. These novel approaches in drug delivery were first used to bind antineoplastic and antibiotic drugs to CNTs for cancer and infection treatment, respectively $[50,51]$.

\section{Uses in Anti-Cancer Treatment}

Many anti-cancer drugs, such as epirubicin, doxorubicin, cisplatin, methotrexate, quercetin, and paclitaxel, have been conjugated with functionalized CNTs and successfully assessed both in vitro and in vivo [52]. The chemotherapy agents can be bound to a complex formed by CNT and antibody against antigen overexpressed on the cancerous cell 
surface. In this way, it is possible to carry the drug mostly at the level of the tumor cells. Drugs can be linked with a magnetic CNT complex, obtained by fixing a layer of magnetite $(\mathrm{Fe} 3 \mathrm{O} 4)$ nanoparticle on the surface of the nanotubes. In this case, an externally placed magnet to target a desired organ interested by the cancer cell localization sparing normal counterparts [53] can guide the system CNT drugs.

Moreover, due to their tiny size and accessible external modifications, CNTs are able to cross the blood-brain barrier (BBB) by various mechanisms targeting for acting as effective delivery carriers to treat brain tumors [54]. CNTs showed some peculiarity in targeting the drug in different tumor types. Taghdisi et al., reported that a tertiary complex of Sgc8c aptamer, daunorubicin, and SWCNT can be internalized effectively into human $\mathrm{T}$ cell leukemia cells (MOLT-4 cells) [55]. A water-soluble SWCNT-Paclitaxel (PTX) conjugate has been found to be highly efficient in suppressing tumor growth when compared with free taxol in a murine 4T1 breast cancer cell model, likely for both the extended blood circulation and enhanced permeability and retention (EPR) effect by SWCNT [56]. Ji et al. developed a highly effective drug delivery system (DDS) based on chitosan and folic acid modified SWCNTs for controllable loading/release of the anticancer agent doxorubicin. The obtained DDS not only effectively killed the hepatocellular carcinoma SMMC-7721 cell lines and depressed the growth of liver cancer but also displayed lesser in vivo toxicity than free doxorubicin. Some studies have demonstrated that CNTs used as carriers can be effectively applied in antitumor immunotherapy [57].

These approaches are based on stimulation of the patient's immune system in order to hit malignant tumor cells. This stimulation can be achieved by the administration of a cancer vaccine or a therapeutic antibody used as drug. In vitro, the conjugation of CNTs with tumor immunogens can activate immune effector T cells; this action is due to the high avidity of antigen on the surface and to the negative charge of CNTs. In this way, it is possible to administer inert materials (CNTs conjugated to tumor antigens) to the patients avoiding the administration of biological material increasing both risks and costs linked to the methods of preparation of the immunogens [58].

\section{Gene Therapy, Drug Delivery}

The potential for SWCNT to act as carriers for small molecules in biological systems is one application that has attracted much attention from academia and industrial research departments for a number of years [59]. It has been found that SWCNT can have low cytotoxicity in some cases and due to their large specific surface area they can carry more active drug molecules per weight unit compared to vesicles and other vehicles typically used. In a scenario where SWCNT are used to carry genes, they have been observed to be able to pass the cell membrane and deliver the gene in the interior of the cell and in the nucleus. Much of the research in different variations of CNT based drug delivery systems is targeted at cancer therapy $[60,61]$. Since much remains unknown about the transport mechanisms, fate and adverse effects of CNT at the cell level applications in the biomedical area are believed not to reach the market in the near future [62].

\section{Organ Toxicity of Medical CNTs}

Respiratory system. One of the most important doors and target organs for CNT is the respiratory system and one of the most widespread routes of human exposure to airborne CNT is inhalation in the workplace and the environment (Fig. 4). Respiratory exposure to CNT can cause important adverse respiratory effects, such as multifocal granulomas, peribronchial inflammation, progressive interstitial fibrosis, chronic inflammatory responses, collagen deposition, pleural lesions and gene mutations, at least in experimental animal studies. The deposition of CNT in the respiratory tract is largely determined by the particle aerodynamic or thermodynamic diameter that gives the probability to the NP to reach deep alveoli that have a size of approximately $20 \mathrm{~nm}$. Therefore, although composed of the same carbon-carbon bond arrangement, Mercer et al. found large differences in the distribution, toxicity and clearance rates of the CNTs from the lungs, depending on the size of their structures [63, 64].

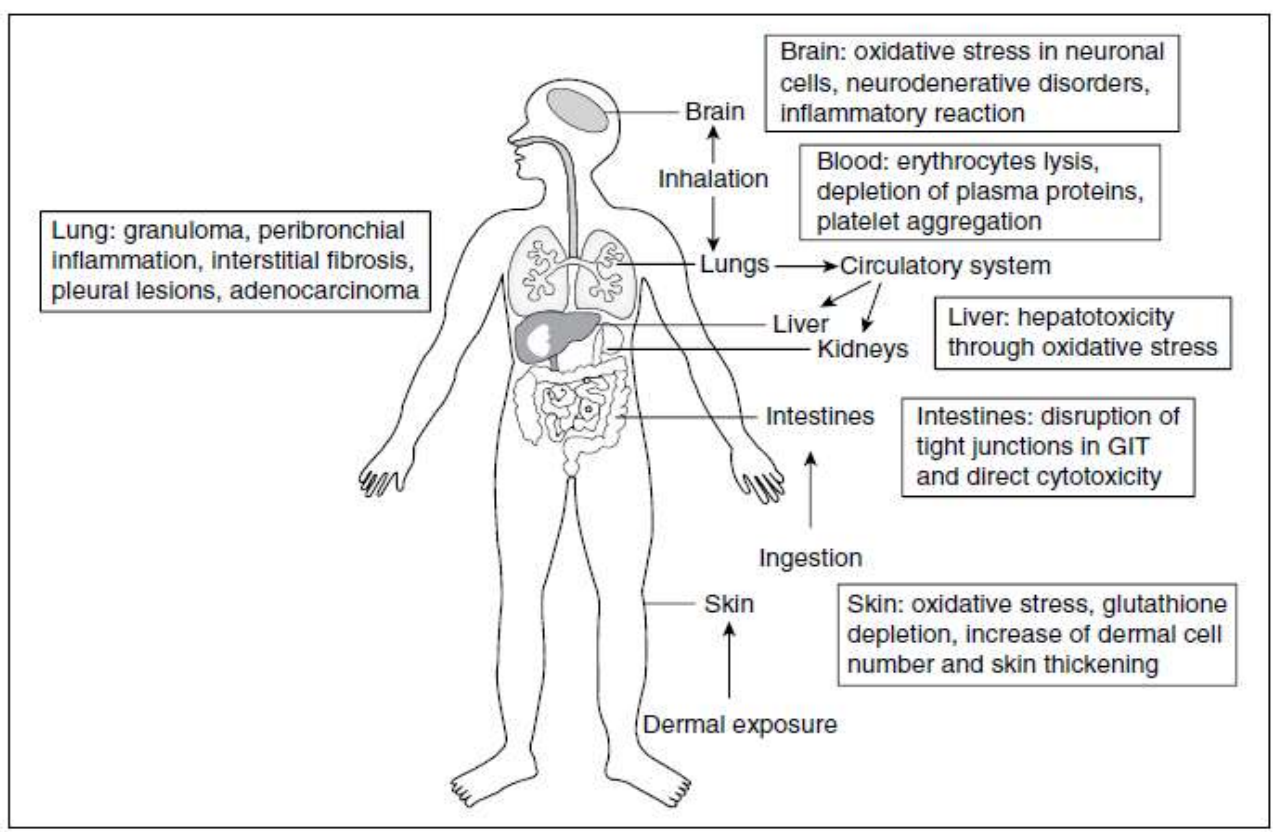

Figure 4: Illustration of the pathways of exposure to carbon nanotubes (CNTs) and associated adverse effects. CNTs can be internalized in cells by inhalation, ingestion, or dermal exposure. Inhaled nanodrugs can pass through epithelia of the respiratory tract into the interstitium and access the bloodstream directly or via lymphatic pathways. Successively, the bloodstream transport nanodrugs to the central nervous system, liver, kidneys, and other organs. Moreover, they can be directly ingested or alternatively, inhaled CNTs can also arrive in gastrointestinal tract. Once CNTs are internalized in cells, they can induce organ-specific toxicity. 


\section{Conclusions}

The use of nanotechnology in medicine is expected to spread rapidly. In recent years, the pharmaceutical industry has used nanoparticles to reduce toxicity and side effects of drugs.[65, 66]. Carbon nanotubes have become common today in global medical research, where scientific research has focused on drug delivery specialties, biosensor methods for disease treatment and health control. Carbon nanotubes have shown that they have the ability to change and transform the process of drug delivery and biological diffusion methods for what is better and better than the situation nowadays. Hence, carbon nanotubes have received the focus of attention in different fields of medicine. The single-walled carbon nanotubes have proven functional because they are able to increase solubility and allow the ability to target tumors or adequately deliver the drug. The functionality also prevents single-walled carbon nanotubes from becoming toxic to cells or altering the function of immune cells. We note that cancer, a group of diseases in which cells grow in an irregular manner, was one of the diseases examined by taking into account how it responded to the drug delivery process by using carbon nanotubes. While we note that today, cancer treatments mainly include surgery, radiotherapy, and chemotherapy. However, it must be borne in mind that these therapeutic methods carry many pain, they are very painful, and they kill many cells in addition to producing multiple side effects $[67,68]$.

\section{References}

1. Wagner FE, Haslbeck S, Stievano L, Calogero S, Pankhurst QA, Martinek KP. (2000) Before striking gold in gold-ruby glass. Nature, 407, 691.

2. Lamberti M, Zappavigna S, Sannolo N et al. (2014) Advantages and risks of nanotechnologies in cancer patients and occupationally exposed workers. Expert Opinion on Drug Delivery. 11: 1087-1101.

3. Caraglia M, Marra M, Misso G et al. (2012) Tumourspecific uptake of anti-cancer drugs: the future is here. Current Drug Metabolism 13: 4-21.

4. Wang X, Wang Y, Chen ZG et al. (2009) Advances of cancer therapy by nanotechnology. Cancer Research and Treatment. 41: $1-11$.

5. Franks A. (1987) Nanotechnology. J Phys E, 20, 1442.

6. Taniguchi N. (1974) On the basic concept of 'nano-technology'. Proceedings of the International Conference on Production Engineering, Tokyo, Japan, Part II.

7. Whitesides, G. M. (2003) The 'right' size in nanobiotechnology. Nat. Biotech. 21, 1161-1165.

8. Lowe, C. R. (2000) Nanobiotechnology: the fabrication and applications of chemical and biological nanostructures. Curr. Opin. Chem. Biol. 10, 428-434.

9. Wang, L.; Zhao, W. and Tan, W. (2008) Bioconjugated silica nanoparticles: development and applications. Nano Res. 1, 99115.

10. Sharma A, Madhunapantula SV and Robertson GP. (2012) Toxicological considerations when creating nanoparticle-based drugs and drug delivery systems. Expert Opinion on Drug Metabolism \& Toxicology. 8: 47-69.

11. Keld Alstrup Jensen, (2015) Senior Researcher, Carbon nanotubes.

12. Mattson M.P., Haddon R.C. and Rao A.M. (2000) Molecular functionalization of carbon nanotubes and use as substrates for neuronal growth. J. Mol. Neurosci. 14 175-82.

13. S. Iijima, T. Ichihashi. (1993) Single-shell carbon nanotubes of 1nm diameter, Nature 363, 603-605.

14. Ajayan PM, Ebbesen TW. (1997) Nanometre-size tubes of carbon. Rep Prog Phys. 60(10):1025.
15. Chico L, Crespi VH, Benedict LX, Louie SG, Cohen ML. (1996) Pure carbon nanoscale devices: nanotube heterojunctions. Phys Rev Lett. 76(6):971-974.

16. M.S. Dresselhaus, G. Dresselhaus, P.C. Eklund. (1995) Science of Fullerenes and Carbon Nanotubes.

17. N. Wang, Z.K. Tang, G.D. Li, J.S. Chen. (2000) Single-walled 4 A carbon nanotube arrays, Nature. 408, 50-51.

18. S. Iijima, (1991) Helical microtubules of graphite carbon, Nature. 354, 56-58.

19. M. Monthioux, L. Noé, L. Dussault, J.-C. Dupin, N. Latorre, T. Ubieto, E. Romeo, C. Royo, A. Monzón and C. Guimon, (2007) Texturising and structurising mechanisms of carbon nanofilament during growth, J. Mater. Chem., 17, 4611-4618.

20. Yu MF, Lourie O, Dyer MJ, Moloni K, Kelly TF, Ruoff RS. (2000) Strength and breaking mechanism of multiwalled carbon nanotubes under tensile load. Science, 287, 637.

21. Ruoff RS, Tersoff J, Lorents DC, Subramoney S, Chan B. (1993) Radial deformation of carbon nanotubes by van der Waals forces. Nature, 364, 514.

22. Palaci I, Fedrigo S, Brune H, Klinke C, Chen M, Riedo E. (2005) Radial elasticity of multiwalled carbon nanotubes. Phys Rev Lett, 94, 175502.

23. Yang YH, Li WZ. (2011) Radial elasticity of single-walled carbon nanotube measured by atomic force microscopy. Appl Phys Lett, 98, 041901.

24. Minary-Jolandan M, Yu MF. (2008) Reversible radial deformation up to the complete flattening of carbon nanotubes in Nano indentation. J Appl Phys, 103, 073516.

25. Ruoff RS, Lorents DC. (1995) Mechanical and thermal properties of carbon nanotubes. Carbon, 33, 925.

26. Tersoff J. (1992) Energies of fullerenes. Phys Rev B, 46, 15546.

27. Falvo MR, Clary GJ, Taylor RM 2nd, Chi V, Brooks FP Jr, Washburn S, Superfine R. (1997) Bending and buckling of carbon nanotubes under large strain. Nature, 389, 582.

28. Wang ZL, Gao RP, Poncharal P, de Heer WA, Dai ZR, Pan ZW. (2001) Mechanical and electrostatic properties of carbon nanotubes and nanowires. Mater Sci Eng C, 16, 3 .

29. Demczyk BG, Wang YM, Cumings J, Hetman M, Han W, Zettl A, Ritchie RO. (2002) Direct mechanical measurement of the tensile strength and elastic modulus of multiwalled carbon nanotubes. Mater Sci Eng A, 334, 173.

30. Zhu YQ, Sekine T, Kobayashi T, Takazawa E, Terrones M, Terrones H. (1998) Collapsing carbon nanotubes and diamond formation under shock waves. Chem Phys Lett, 287, 689.

31. Sinnott SB, Shenderova OA, White CT, Brenner DW. (1998) Mechanical properties of nanotubule fibers and composites determined from theoretical calculations and simulations. Carbon, 36, 1 .

32. Yakobson BI. (1998) Mechanical relaxation and "intramolecular plasticity" in carbon nanotubes. Appl Phys Lett, 72, 918.

33. Ru CQ. (2000) Effect of van der Waals forces on axial buckling of a double-walled carbon nanotube. J Appl Phys, 87, 7227.

34. Guanghua G, Tahir C, William AG, III. (1998) Energetics, structure, mechanical and vibrational properties of single-walled carbon nanotubes. Nanotechnology, 9, 184.

35. Hernandez E, Goze C, Bernier P, Rubio A. (1998) Elastic properties of $\mathrm{C}$ and $\mathrm{BxCyNz}$ composite nanotubes. Phys Rev Lett, $80,4502$.

36. Akiladevi D, Basak S. (2010) Carbon nanotubes (CNTs) production, characterization and its applications. Int J Adv Pharm Sci, $1,187$.

37. Banerjee S, Naha S, Puri IK. (2008) Molecular simulation of the carbon nanotube growth mode during catalytic synthesis. Appl Phys Lett. 92(23):233121. 
38. Rao CNR, Cheetham AK. (2006) The Chemistry of Nanomaterials: Synthesis, Propertiesand Applications. 1st edition. Oxford University: John Wiley \& Sons.

39. Hou P, Liu C, Tong Y, Xu S, Liu M, Cheng H. (2001) Purification of single-walled carbon nanotubes synthesized by the hydrogen arc-discharge method. J Mater Res. 16(09):2526-2529.

40. Mizoguti E, Nihey F, Yudasaka M, Iijima S, Ichihashi T, Nakamura K. (2000) Purification of single-wall carbon nanotubes by using ultrafine gold particles. Chem Phys Lett. 321(3):297301.

41. Huang X, Mclean RS, Zheng M. (2005) High-resolution length sorting and purification of DNA-wrapped carbon nanotubes by size-exclusion chromatography. Anal Chem. 77(19):6225-6228.

42. Hersam MC. (2008) Progress towards monodisperse singlewalled carbon nanotubes. Nat Nanotechnol. 3(7):387-394.

43. Gu Z, Peng H, Hauge RH, Smalley RE, Margrave JL(2002) Cutting single-wall carbon nanotubes through fluorination. Nano Lett. 2(9):1009-1013.

44. Popov VN. (2004) Carbon nanotubes: properties and application. Materials Science and Engineering: R: Reports. 43(3):61-102.

45. Baughman RH, Zakhidov AA, de Heer WA. (2002) Carbon nanotubes - the route toward applications. Science. 297(5582):787-792.

46. Terrones M. (2003) Science and technology of the twenty-first century: synthesis, properties, and applications of carbon nanotubes. Annu Rev Mater Res. 33(1):419-501.

47. Ye X, Lin Y, Wang C, Wai CM. (2003) Supercritical fluid fabrication of metal nanowires and nanorods templated by multiwalled carbon nanotubes. Adv Mater. 15(4):316-319.

48. Wu HQ, Wei XW, Shao MW, Gu JS. (2004) Synthesis of zinc oxide nanorods using carbon nanotubes as templates. J Cryst Growth. 265(1):184-189.

49. Hirlekar R, Yamagar M, Garse H et al. (2009) Carbon nanotubes and its applications: a review. Asian Journal of Pharmaceutical and Clinical Research. 2: 17-27.

50. Lamberti M, Zappavigna S, Sannolo N et al. (2014) Advantages and risks of nanotechnologies in cancer patients and occupationally exposed workers. Expert Opinion on Drug Delivery. 11: 1087-1101.

51. Patole SP, Alegaonkar PS, Lee HC et al. (2008) Optimization of water assisted chemical vapor deposition parameters for super growth of carbon nanotubes. Carbon. 46: 1987-1993.

52. Lamberti M, Zappavigna S, Sannolo N et al. (2014) Advantages and risks of nanotechnologies in cancer patients and occupationally exposed workers. Expert Opinion on Drug Delivery. 11: 1087-1101.

53. Masotti A and Caporali A. (2013) Preparation of magnetic carbon nanotubes (Mag-CNTs) for biomedical and biotechnological applications. International Journal of Molecular Science. 14: 24619-24642.
54. Caraglia M, De Rosa G, Salzano G, et al. (2012) Nanotech revolution for the anti-cancer drug delivery through blood-brain barrier. Current Cancer Drug Targets. 12: 186-196.

55. Taghdisi SM, Lavaee P, Ramezani M et al. (2011) Reversible targeting and controlled release delivery of daunorubicin to cancer cells by aptamer-wrapped carbon nanotubes. European Journal of Pharmaceutics and Bio pharmaceutics. 77: 200-206.

56. Liu Z, Chen K, Davis C et al. (2008) Drug delivery with carbon nanotubes for in vivo cancer treatment. Cancer Research. 6(8): 6652-6660.

57. Ji Z, Lin G, Lu Q et al. (2012) Targeted therapy of SMMC-7721 liver cancer in vitro and in vivo with carbon nanotubes based drug delivery system. Journal of Colloid Interface Science. 365: 143149.

58. Gottardi R and Douradinha B. (2013) Carbon nanotubes as a novel tool for vaccination against infectious diseases and cancer. Journal of Nano biotechnology. 11: 30.

59. C. R. Martin and P. Kohli, (2003) The emerging field of nanotube biotechnology. Nature Reviews Drug Discovery. 2(1), 29-37.

60. N. W. S. Kam, M. O'Connell, J. A. Wisdom, et al., (2005) Carbon nanotubes as multifunctional biological transporters and near infrared agents for selective cancer cell destruction. Proceedings of the National Academy of Sciences of the United States of America. 102(33), 11600-11605.

61. P. C. Lee, Y. C. Chiou, J. M. Wong, et al., (2013) Targeting colorectal cancer cells with single-walled carbon nanotubes conjugated to anticancer agent SN-38 and EGFR antibody. Biomaterials. 34(34), 8756-8765.

62. D. M. Guldi and M. Nazario, (2010) carbon nanotubes and related structures - synthesis, characterization, functionalization, and applications, Wiley-VCH Verlag GmbH \& Co. KGaA, Weinheim, Weinheim, 1-539.

63. Ferreira AJ, Cemlyn-Jones J and Robalo Cordeiro C. (2013) Nanoparticles, nanotechnology and pulmonary nanotoxicology. Revista Portuguesa de Pneumologia. 19: 28-37.

64. Wang L, Stueckle TA, Mishra A et al. (2014) Neoplastic-like transformation effect of single-walled and multi-walled carbon nanotubes compared to asbestos on human lung small airway epithelial cells. Nano toxicology. 8: 485-507.

65. Lamberti M, Zappavigna S, Sannolo N et al. (2014) Advantages and risks of nanotechnologies in cancer patients and occupationally exposed workers. Expert Opinion on Drug Delivery. 11: 1087-1101.

66. Caraglia M, Marra M, Misso G et al. (2012) Tumourspecific uptake of anti-cancer drugs: the future is here. Current Drug Metabolism. 13: 4-21.

67. C. Srinivasan, (2008) Carbon nanotubes in cancer therapy. Current Science. 94, 300.

68. T. A. Hilder, J. M. Hill, (2008) Carbon nanotubes as drug delivery Nano capsules. Current Applied Physics. 8, 258. 